\title{
Recommendations for the Sharing Economy Safeguarding Privacy
}

\author{
Ranzini, Giulia; Kusber, Nina; Vermeulen, Ivar; Etter, Michael
}

Document Version

Final published version

DOI:

$10.2139 /$ ssrn.3107525

Publication date:

2018

License

Unspecified

Citation for published version (APA):

Ranzini, G., Kusber, N., Vermeulen, I., \& Etter, M. (2018). Recommendations for the Sharing Economy:

Safeguarding Privacy. SSRN: Social Science Research Network. https://doi.org/10.2139/ssrn.3107525

Link to publication in CBS Research Portal

\section{General rights}

Copyright and moral rights for the publications made accessible in the public portal are retained by the authors and/or other copyright owners and it is a condition of accessing publications that users recognise and abide by the legal requirements associated with these rights.

\section{Take down policy}

If you believe that this document breaches copyright please contact us (research.lib@cbs.dk) providing details, and we will remove access to the work immediately and investigate your claim. 
Report from the EU H2020 Research Project Ps2Share:

Participation, Privacy, and Power in the Sharing Economy

\section{Recommendations for the Sharing Economy: Safeguarding Privacy}

Giulia Ranzini ${ }^{1}$, Nina Kusber ${ }^{2}$, Ivar Vermeulen ${ }^{1}$, and Michael Etter ${ }^{3}$

${ }^{1}$ Vrije Universiteit Amsterdam

${ }^{2}$ JoVoto, Berlin

${ }^{3}$ Copenhagen Business School 


\section{Introduction: Privacy in the Sharing Economy}

This report, 'Recommendations: Privacy', forms one element of a European Union Horizon 2020 Research Project on the sharing economy: Ps2Share 'Participation, Privacy, and Power in the Sharing Economy'. The study is undertaken within the scope of the European Union's Horizon 2020 research and innovation programme, funded under grant agreement No. 732117 and with the objective (ICT35) of "Enabling responsible ICT-related research and innovation".

This project aims to foster better awareness of the consequences which the sharing economy has on the way people behave, think, interact, and socialize across Europe. Our over-arching objective is to identify key challenges of the sharing economy and improve Europe's digital services through providing recommendations to Europe's institutions. We focus on topics of participation, privacy, and power in the sharing economy.

The project is comprised of four primary tasks: A series of three reviews of the existing literature on the sharing economy (Andreotti, Anselmi, Eichhorn, Hoffmann, \& Micheli, 2017; Newlands, Lutz, \& Fieseler, 2017a; Ranzini, Etter, Lutz, \& Vermeulen, 2017) an analysis of platforms operating within Europe (Stanoevska-Slabeva, Lenz-Kesekamp, \& Suter, 2017; Stanoevska-Slabeva, Lenz-Kesekamp, \& Suter, 2018), a series of focus groups among 'millennials' within 5 European countries (Ranzini, Newlands, Anselmi, Andreotti, Eichhorn, Etter, Hoffmann, Jürss, \& Lutz, 2017), and a representative survey of more than 6000 citizens across 12 European countries (Andreotti, Anselmi, Eichhorn, Hoffmann, Jürss, \& Micheli, 2017a; Newlands, Lutz, \& Fieseler, 2017b; Ranzini, Etter, \& Vermeulen, 2017). This report provides a set of summary recommendations for the various stakeholders of the sharing economy. Recommendations are provided for users (both providers and consumers), sharing economy platforms, educators, and policy makers. Similarly, two additional reports provide recommendations for different stakeholders in terms of participation (Andreotti, Anselmi, Eichhorn, Hoffmann, Jürss, \& Micheli, 2018) and power (Newlands, Lutz, \& Fieseler, 2018). 


\section{Executive Summary}

In the following document, we wish to build on our research to provide recommendations for all actors involved in the sharing economy: Users, both acting as consumers and as providers, platforms, policy makers, and educators. For each group of actors, we provide five main recommendations.

When it comes to providers within the sharing economy, we recommend that providers should:

1. Be aware of their own data rights, for example by staying informed about privacy policy changes and new regulations.

2. Respect online privacy (own and other users') by thinking critically about their own information sharing and respecting the boundaries set by consumers.

3. Set their own boundaries, especially when spaces are shared, which might mean including stricter guidelines or being very clear on physical boundaries.

4. Write honest reviews, keeping in mind what can be useful for other providers.

5. Protect the privacy of third parties, such as friends and family who might still be involved with the properties being shared.

Consumers of the sharing economy face some of the same challenges, but have additional vulnerabilities. We have five main recommendations for them, where consumers should:

1. Be critical and respectful around private information, that is, consumers should think of the consequences when sharing their own data and accessing the data of others.

2. Respect providers' online privacy, avoiding, for example, sharing their personal details on social media or other platforms.

3. Respect providers' offline boundaries by following providers' rules about the limits to their shared spaces.

4. Review honestly, rate appropriately, both when faced with a positive and a negative experience.

5. Approach off-platform offers with caution, that is, considering the advantages that might appear evident alongside the additional risks, especially concerning privacy and fraud.

The results to our project highlight that sharing economy platforms have important responsibilities when it comes to privacy. We summarized our recommendations in five main suggestions, where sharing economy platforms should:

1. Embrace more transparency by providing more insights around what data is collected, how it is analysed, and how long it is stored

2. Set service standards, specifying, for example, what responsibilities should be covered by each role for each type of service. 
3. Improve feedback systems, providing guidelines for what should be included in reviews and minimizing the impact of positive biases.

4. Protect vulnerable users by embracing diversity among providers and setting out policies aimed at minimizing the risks to minorities.

5. Approach privacy as an opportunity to diversify, considering how different services could be offered to the most privacy concerned users.

Educators have an important role in supporting the active, safe, and enthusiastic participation of users in the sharing economy. Below are five recommendations for teachers and educational institutions, which should:

1. Prioritize digital literacy, especially focusing on younger users and mobile skills.

2. Focus on users' rights, informing the public about evolutions, opportunities, and threats.

3. Promote safer participation by encouraging users to think critically about the level of information sharing that feels safest for them.

4. Approach local differences by adapting education efforts to local variables, such as technology diffusion, or local services.

5. Lead by example by promoting a culture of sharing within educational institutions.

Our final recommendations are for policy makers, who have an important responsibility in making the sharing economy healthy and sustainable. Our five privacy-related recommendations are the following, where policy makers should:

1. Regulate data collection by platforms by setting limits on what data should be collected and homogenizing regulation on server storage.

2. Deal with internationality by approaching the challenges resulting from the multi-nationality of sharing platforms.

3. Deal with complexity by approaching platforms for all their offered services, including intermediaries.

4. Encourage communication and determine responsibilities by promoting clear information exchanges between users and platforms and defining who is responsible in case of misconduct.

5. Regulate data collection by peers by establishing limits to the kind of information that can be collected, and determining how and how long it should be stored. 


\section{Recommendations for Providers}

\section{Providers should be aware of their data rights}

Providers of sharing services are at the center of multiple data exchanges:

- On the one hand, they share their information with platforms in exchange for access

- ...on the other hand, they receive information from consumers, which is then used to choose who gets to participate in the exchange.

As such, providers are vulnerable to breaches of their own data, as well as of data of third parties. Providers should be particularly proactive so as to minimize risks as much as possible. It is therefore absolutely a priority for providers to focus on existing rules around data. In particular, we think they should focus on the following aspects of data collection and storage:

- What changes are brought to privacy settings on the platform, and what consequences they could have for the service offered to consumers?

- What type of data can providers collect about consumers? For example, could a ride-sharing driver ask for the phone number of a passenger who is not their primary contact? Could a home-sharing host ask for the passport of a guest?

- How such data should be stored (e.g., digitally, or in a physical copy) and how long it should be kept?

We think that keeping updated on data rights helps providers to evaluate whether access to the platforms justifies the risks and possible sense of vulnerability emerging from sharing their data. Reflecting on the collection and storage of consumers' data also reminds providers of their rights and duties around the data of others, potentially making their participation more informed and responsible.

Additionally, if the privacy calculus of providers becomes an explicit process, in that they think carefully of what benefits they derive from sharing their own data, they are less likely to perceive data provision as inevitable. This can lead providers to more conscious participation, in which roles are better defined and alternative options are sought, should they not feel comfortable with the amount of shared data. 

spected)

Providers of sharing economy services have access to a substantial amount of information from consumers, such as their full names, phone numbers, and sometimes social media accounts. While personal interactions are often an outcome of sharing economy interactions, providers should be aware that their role towards other users remains largely professional. As such, we recommend that they respect consumers' privacy, for example by adopting the following behaviors:

- Asking for consent before taking any action that transcends a merely professional relationship, such as adding a consumer on their social media.

- Not using pictures of guests or passengers for promotional or other purposes.

- If additional consumer information is necessary, for example because local regulations apply, clear explanations should be provided for the reasons.

Additionally, because the existing feedback system might influence users' behavior towards what could generate more positive reviews, we suggest that providers keep a professional attitude around data exchanges with consumers at least until their transaction is finished. This also helps providers set clear boundaries around how consumers should behave, and what actions are considered inappropriate or unacceptable.

Aside from the duty to take care of consumers' privacy, providers should also take measures to protect their own data. When it comes to offline interactions, they should feel free to set their own boundaries around consumer access to their private spaces. Speaking of online information sharing, they should strive to obtain a level of information disclosure that makes them feel comfortable.

In fact, providers find themselves balancing two types of incentives:

- On the one hand, the provision of additional information improves the chance to exclude consumers who might not be a good match, and makes a provider's profile overall more attractive

- ...on the other hand, more information makes providers more identifiable, and potentially more vulnerable in terms of privacy.

As such, providers should weigh the opportunities stemming from providing additional information against the potential risks and make, as much as possible, an informed decision around their desired level of information disclosure. 


\section{Providers should set their own boundaries}

The establishment of limits becomes even more important when providers get to actually share their own goods, accommodation, or transportation with consumers. In order to minimize the chances of a violation of boundaries or of an unintentional breach of physical privacy, we recommend that providers establish and communicate clear rules about how their shared property should be used.

Especially when sharing accommodation, we would recommend that providers consider protecting the goods they care about, either by removing them from the spaces that will be shared with guests, or by covering them with additional insurance. Part of the process of setting boundaries involves excluding that important objects and property get damaged or destroyed while being temporarily used by consumers.

In all instances of sharing of private spaces, providers should be explicit about the behaviors consumers should not exhibit while using the shared property. We recommend that hard limits are communicated openly on their public profiles, rather than privately or on an individual level. This not only helps providing a clear ground for eventual claims, but makes sure that consumers self-select in response of the profile they see, and engage in the exchange knowing exactly what they are not supposed to do. We believe that providers have the right to choose who to share their property with, and this is really only possible if full information about what they expect is provided.

Limits should also be provided when it comes to provider-consumer interactions. The social side to the sharing economy is an important feature of most platforms and is what leads many users to pick sharing services over traditional alternatives. However, it can lead consumers to expect instructions, dialogue and in some cases entertainment from providers, which might lead to uncomfortable situations. Also in this case, it helps to clearly communicate limits, for example a ride-sharing driver who prefers to not talk might find ways to signal it to consumers, and a host could provide time limits during which they could be contacted.

Setting clear boundaries helps providers identify and sanction consumers' behavior perceived as privacy-invasive. We recommend that providers get acquainted with what procedures to follow in case of a privacy breach, both with the platform and with relevant authorities. Ultimately, it is wise for providers to trust their feelings around behaviors they perceive as inappropriate, and signal them to their sharing platform of reference. 


\section{Providers should write honest reviews about what matters}

Writing negative reviews can be a challenging process on both sides of the sharing economy, as users might find themselves limiting their criticism out of concerns around their own reputation. In particular, for providers, unjust criticism from a mass of consumers can result in their own exclusion from participating on the platform. This might lead providers to tread carefully around their consumer reviews and minimize problems that might have emerged in the interaction.

While we recognize that some of the issues around the positivity bias of reviews should rather be addressed by platforms (see chapter 3.3.3), we recommend that providers attempt, as much as possible, to provide honest reviews. This includes:

- Highlighting what worked and did not work in the interaction with the specific consumer.

- Providing information over whether any of the set rules were violated.

- Providing context for eventual criticisms (was there any justification for the behavior of consumers? Was it a single instance of inappropriate behavior or were there violations throughout the interaction?).

Such information helps other providers make their own evaluation around whether they should accept or reject an offer from a specific consumer.

Whether their reviews might be positive or negative, providers should care about the personal information of consumers. When highlighting positive behavior or criticizing unpleasant interactions, providers should make sure that they do not disclose information (such as surnames, addresses, etc.) that consumers did not disclose themselves on their own profiles. This ensures that a privacy violation does not take place.

Overall, we wish to bring the attention of providers to the helpfulness of writing useful reviews. An ideal structure should include the following elements:

- Information about the timeliness and pleasantness of interaction with the customer.

- Information on whether they perceived to have received fair and respectful treatment for themselves and their goods.

- Information on whether they would recommend that another provider engages in a similar exchange.

This should help in providing a review that is as factual as possible and therefore useful for other providers who might want to engage in similar interactions. 


\section{Providers should not forget about the privacy of those around them}

Because the sharing economy takes place within communities of people, its privacy repercussions do not necessarily only involve those who participate in the exchange. As such, providers should pay attention that the privacy of everyone involved in their lives is preserved, irrespective of whether they take part in their sharing activities.

For providers who share accommodation, precautions should be taken so that other people living on the same premises, be it family members or neighbors, are put in the condition of not interacting with consumers of the sharing services. This can be achieved, for example, by taking the following precautions:

- Communicating to consumers limits on access to the shared property (e.g., should ride-sharing passengers use the front or the backseat? What areas should be accessible to a homesharing guest?)

- Only entering sharing agreements when everyone living or visiting the property is informed and has agreed to the sharing.

- Excluding from the sharing agreement areas of the home or objects that belong to family or roommates.

- Agreeing with roommates or family on dates and times during which sharing should take place, so as to minimize their discomfort.

Providers who share homes or transportation vehicles which they also employ for personal purposes should also ensure that no personal belongings, and especially no photos or similarly identifying objects are left in the vehicles while they are being shared. Similar care should be placed in not displaying phone numbers on phones, or previous addresses on GPS receivers.

Aside from protecting the privacy of local communities, we feel like our recommendations offer a basis for the sustainability of the sharing economy, making it participative for those who want to take part in it, but also respectful of those who are not involved. 


\section{Recommendations for Consumers}

\section{Consumers should be critical and respectful around private data}

For consumers of the sharing economy, maintaining a level of proactivity around personal data sharing on platforms is also recommended. This is motivated by the fact that, much like providers, they exchange data both with the platforms and with peers, therefore exposing themselves to potential privacy violations in multiple contexts.

One way in which consumers can increase their knowledge around what data they can be expected to share is by staying as updated as possible about changes on the platforms' privacy policies. Because such documents are often long and difficult to interpret by the broad public, we recommend that users rather start by setting their privacy preferences as close as possible to what they are comfortable with. This should put them in the condition of:

- Noticing changes and reflecting on whether they add value to their experience of the platforms.

- Eventually making requests to the platforms whenever they feel like options do not match their wished level of protection.

When it comes to peer-related privacy concerns, consumers should stay informed on what type of information they can, and maybe should, share with providers. This might require them to check whether a provider request (for example, a ride-sharing driver asking for a passenger's driver license) conforms with the rules established by the platform. The advantages of staying informed, however, extend beyond consumers' privacy protection. Once consumers know what information providers can request, they can make sure they have all materials available and therefore increase both their likelihood of accessing the service and the ease of the transaction.

Overall, we advocate that consumers keep track of whether the information requested by both platforms and providers matches what they wish to provide, and that they seek out alternatives should this not be the case. When the conditions for data sharing seem acceptable, it is still recommended that consumers keep safety measures in mind and emergency numbers on hand, so that they can minimize both risks and damages. 


\section{Consumers should respect providers' online privacy}

Consumers of the sharing economy should keep in mind that their transactions with providers are commercial in nature. This is even the case when pleasant interactions occur between the two parties. This means that providers, as well as their families, friends, and extended circles, have lives outside of their sharing activities, in which they often use the same homes, cars, or tools. Because of this, consumers should be cautious in sharing information about providers outside of the sharing platforms. In particular, consumers should consider:

- Putting in an effort to avoid sharing or commenting on providers' private information on social media (such as: posting pictures of children and pets, or disclosing family names)

- Using official customer care channels as provided by platforms, including platforms' dedicated social media accounts, before using their personal media for complaints. This insures their safety and minimizes the chance that additional information, about both the consumer and the involved provider, might be put online without their consent.

- Limiting the overall information they post on social media to pictures and details about the shared property, rather than about the providers sharing it.

While interactions through a sharing economy platforms can generate pleasant social experiences, they remain, for the most part, commercial transactions. As such, for as long as the transaction takes place, we recommend that consumers maintain the relationship as being largely professional. Consumers should refrain from adding their providers on social media and should limit the request of information to what is admissible on the platform. By nature of the interaction on a sharing platform, consumers have access to private, and potentially sensitive information of providers, such as home addresses and license plates. They should not use this information for other purpose than the transaction.

Especially for platforms that are more social than commercial in nature, such as hospitality networks, consumers might find themselves wanting to keep in touch with providers, perhaps so as to reciprocate hospitality or maintain a social connection. This can be established, for example, by adding a provider on one's contacts on a social network site. We recommend that users proceed asking each other's permission and respecting providers' consent. 


\section{Consumers should respect providers' physical boundaries (as well as their}

own)

Because sharing economy interactions involve, to a varying extent, the physical presence of providers and consumers within the same space, it is of vital importance that consumers respect as much as possible the rules set by providers when operating in a shared space. This means, for example:

- respecting providers' wishes around behaviors that should be kept throughout the interaction

- refraining from using rooms and tools that might have been excluded from the sharing agreement

- asking for consent before taking pictures (including selfies) while on the sharing property

It could be useful for consumers to keep the traditional alternatives to the sharing economy as a guideline: using a ride-sharing service should entail at least the same level of respectfulness as riding in a traditional taxi. Additionally, because consumers are dealing with the property of another individual, and not that of an organization, they should consider this as an opportunity to ask for guidelines whenever in doubt on the behavior they should keep.

In fact, social interaction is indeed additional value offered by sharing economy services, as compared to their traditional alternatives. Because consumers access the property of a peer, rather than e.g. a room from a hotel chain, they might get to connect to the property's owners and make the interaction more personal and meaningful. Most providers enjoy this part of their roles, and are happy to provide consumers with information, as well as interact with them and make sure their relationship is pleasant. However, such interaction might become a burden if consumers expect entertainment from providers, or if they formulate requests that are beyond their role and potentially invasive of their privacy. Consumers should be aware of this while making requests to providers.

The attention to one's physical boundaries, i.e. the limits to one's physical and emotional comfort, should also be placed in consumers' own experience. In particular, consumers should be careful in the following situations, e.g.

- when a provider has expectations towards them that they disagree or don't feel comfortable with

- when they feel like their privacy or comfort is violated

- when they perceive to be treated unfairly on in a discriminatory manner.

In any of such cases, they should immediately seek support from the sharing platform and/or the authorities. 


\section{Consumers should review honestly, rate appropriately}

Feedback systems are essential to the sharing economy, as they allow for trust to form between consumers and providers, and reward efforts put in positive behavior on both sides. Consumers' reviews of providers offer important information to other consumers, who might decide to trust (or not to trust) the same providers for their required services. As such, consumers should focus their review on information they consider useful for someone who might be in their same position. This should include, for example:

- information about the shared property (e.g. whether a car is wheelchair-friendly)

- information about the providers (e.g. whether they were responsive and pleasant)

- information about their interaction with providers (e.g. whether the ride-share went as planned, whether the hosts were present to hand the keys to an apartment).

It is important to highlight that issues of positive bias can occur also when it comes to consumer reviews. When faced with the necessity to inform other consumers of problems in the interaction with a provider, consumers might be worried about receiving a negative review in return, which could impact their reputation on the platform, and their ability to access it in the future.

We think consumers should feel incentivized to write both positive and negative reviews. While platforms should me mainly responsible to make this happen (see chapter 3.3.3), there are some tips we can provide for consumers, so that their reviews are ultimately useful for other consumers who might be in the position to make similar decisions.

- When feedback can be provided in the form of a review, consumers should try to highlight both the positive and the negative aspects of their experience. Without being over-critical, this exercise helps other consumers make an informed decision.

- When incidents happen, or whenever consumers feel they should provide a negative review, it is helpful to provide as much context as possible.

- Overall, ratings should reflect what is to be expected from a shared property, and not consumers' personal expectations of a service. When in doubt, it can be helpful for consumers to consult the guidelines about what should be expected from a shared property.

When writing reviews, it is also important for consumers to pay attention to how much of providers' information they share. Addresses, phone numbers and other identifying information that is not available on the provider's profile should not be included. 

caution

As consumers interact directly with providers through the platforms, and sometimes continue their conversations through social media or via phone calls/text messages, it can happen that they receive offers for the same service (such as home-sharing or ride-sharing) to take place outside of the platform. Such types of commercial interactions can sound appealing to consumers, who might both save money and avoid sharing data with platforms. They could also perceive similar offers as a sign of mutual trust established with the individual provider. However, an off-platform direct offer also brings about disadvantages and risks of which consumers should be aware.

- Primarily, off-platform commercial interactions happen between users and are therefore not covered by contracts of any sorts or legal protection.

- In the context of privacy, this means that eventual misuse of private data, or breach of personal boundaries, can only be dealt with by engaging in legal measures against a private person.

- Additionally, off-platform exchanges are not covered by any of the insurances provided by platforms for traditional sharing economy interactions, and as such, can make consumers more vulnerable to frauds.

- The inability to rate and review such transactions also makes it more difficult for consumers to warn other consumers of eventual risks and dangers.

Overall, because we recognize a value for consumers in the development of rapport with providers, which could potentially further develop into a fully legal commercial relationship, we suggest caution rather than advising consumers to avoid off-platform transactions at all costs. Consumers should be aware of the increased risks of off-platform transactions, as well as the advantages, and evaluate whether if they feel like they can still guarantee a fair and safe experience for themselves. 


\section{Recommendations for Platforms}

\section{Sharing economy platforms should be critical and respectful around pri- vate data}

Sharing platforms require user data in order to improve the personalization, usefulness, and safety of services. However, users are often unaware of what data is collected, which can lead to feelings of vulnerability and exposure. Fears around systematic privacy invasions, as carried out by sharing platforms, can limit the willingness of users to participate in the sharing economy. As such, we recommend that transparency is treated as a priority by sharing platforms.

In particular, transparency should start with data collection. Sharing platforms should aim to communicate more openly about their collection and storage of data. In particular, we recommend that attention is placed on:

- Communicating clearly how user data is collected, for both consumers and providers, and how long it stays on the platform servers.

- Providing instruments to users to check the platform claims, for example by requesting a copy of all their data that is in possession of the platform.

Another theme on which more transparency should be achieved is platforms' use of algorithms around users' profiles. While it is understandable that platforms might want to preserve some level of secrecy over their algorithms in order to stay competitive, they should strive for more openness at least when it comes to:

- The rules behind profile placement for providers - explaining, for example, whether higher availability leads to a more visible placement.

- What regulates the matching of providers with consumers - being more open on this aspect could lead to a more efficient information disclosure by both parties.

One last theme on which platforms could achieve more transparency is the communication of instruments available to both providers and consumers in the case of a privacy breach. This should not be limited, as sometimes happens in practice, to numbers to call in case of fraud, but should rather cover both online and offline privacy breaches. Some examples include:

- Guidelines on what to do in case of suspected hacking or identity theft.

- Guarantee to act on provider and consumer profiles reported as fraudulent.

- Immediate assistance in case of physical treats, boundary breaches, or other kinds of inappropriate behavior by both providers and consumers. 


\section{Sharing economy platforms should set service standards}

In order to function properly, sharing platforms require the collaboration and participation of both consumers and providers. Such active presences become difficult if definitions for roles are not provided or not sufficiently clear:

- For providers, not knowing what to include in their service could lead to significant differences in the offer, leading to unsustainable competition.

- For consumers, not knowing what should be included in a service received could lead to excessive or unreasonable expectations, which could damage the trust mechanisms behind feedback systems and, in turn, the functioning of platforms.

As such, we recommend that sharing platforms set more precise service standards, clearly delimitating the roles and responsibilities of both providers and consumers to the sharing economy.

For providers, service standards should include:

- What they should include within their services (e.g., should cleaning be within the offer for a shared apartment? Should food or beverages be part of ride-sharing?).

- What type of interactions could be expected by consumers.

- What additional services can, but do not have to be offered.

- What consumers should not expect from providers, under any circumstance.

For consumers, service standards should include:

- What they should expect from a provider, and what is fair to request.

- What type of interactions they should expect from a provider, and what can be expected from them.

- What is 'good' consumer behavior.

- What providers should not expect from consumers, under any circumstance.

Rules should exist around the management of data from other users. This should cover both phases of the provider-consumer interaction:

- While interacting on the sharing platform, both categories of users should be aware of the limits to data collection and know what additional information can be requested from providers/consumers.

- Outside of sharing platform interaction, providers especially should be informed of how to store data from consumers and how long they should keep it. 


\section{Sharing economy platforms should improve feedback systems}

A well-functioning feedback system, where reviews of providers and consumers are timely and verifiable, provides an important backbone to the functioning of sharing platforms and helps to incentivize motivated and enthusiastic participation. As such, we recommend that platforms prioritize their feedback system and put efforts into making it efficient and sustainable.

A first intervention we suggest to platforms is that of providing clear communication on how users should be rated, and what should be included in reviews. In practice, this means:

- Providing guidelines on what level of services (or the levels of consumer behaviors) should be associated with every rating grade (e.g., stars). For example, a matrix of suggestions could help both consumers and providers base their ratings on a concrete set of references, rather than exclusively on perceptions or feelings.

- Including suggestions (such as ideal structures, or full examples) on what information should be included in reviews. This gives both providers and consumers the opportunity to contribute reviews that are useful for the community, focusing on what can help other users make an informed decision.

One of the inherent risks of reviewing, especially when a review from both sides of an interaction is required, is a degree of positive bias. This means that users will publish a review that is more positive than their actual experience in the fear that, should they share their negative experience, they might receive a negative review in return and suffer negative reputational consequences. This mechanism decreases the trustworthiness of the whole feedback system, leading users to doubt the validity of reviews they read, especially if they are positive.

Platforms could minimize the chances of positive bias influencing reviews by:

- Structuring reviews around specific guidelines, so that a criticism about a specific aspect of the individual consumer or provider (e.g., cleanliness) does not translate into a bad overall evaluation.

- Providing opportunities for users to provide unpublished and eventually anonymous feedback directly to the platform.

In addition to the above, both providers and consumers should be made aware that in case of inappropriate behaviors by the other party, reviews are not an appropriate tool for sending complaints. Rather, other measures should be put in place. Platforms should make it a priority to respond quickly and efficiently to such claims. 


\section{Sharing economy platforms should protect vulnerable users}

Even when making an effort to provide a fair and equal experience to all users, sharing economy platforms operate internationally and thus face a number of challenges concerning the potential discrimination of users, based on their gender, age, or minority status. While platforms should be committed to minimize any form of stereotyping, bias, or discrimination, their efforts might not be sufficient.

First of all, we think it is important for platforms to realize that some categories of users might face additional privacy threats or have additional concerns in the online and offline interactions with other users. Such specific risks could include:

- Being rejected by a provider or being unsuccessful with consumers.

- Being stalked or having private information disclosed online.

- Being intimidated, molested, or sexually harassed.

Platforms should be aware of the vulnerabilities of specific categories of users and do what is possible to protect them and prevent incidents from happening. This can be achieved through a series of interventions. Below are some examples:

- Promote diversity: Encourage the participation of individuals from all ethnical backgrounds, genders, and sexual orientations both as consumers and providers. This could improve the perception of safety on both sides (for example: female consumers might be more motivated to accept a ride-share with a female provider).

- Start a conversation with providers: Home- and ride-sharers might have preferences around which consumers they want to cater to. For example, an older couple might prefer to host families rather than groups of young travelers. A single woman might prefer hosting other women. However, categorically excluding categories of consumers can hide racial or sexist undertones. We think that platforms should start a conversation with providers, in the hopes of gaining a better understanding of where limits should be set on personal choice.

- Discourage and punish inappropriate behavior: platforms should provide users with dedicated channels to report discriminating, harassing, or violent behavior. Consumers and providers acting inappropriately should be immediately excluded from further participation within the platform. Platforms should pay particular attention that the safety and wellbeing of minorities is guaranteed and protected. 

to diversify

Not all users of the sharing economy present similar levels of concern when it comes to their privacy. This gives them very different expectations towards the services they find on platforms. This means that, while some users are very comfortable with the existing levels of privacy protection, both in case of invasion by peers or by the platforms themselves, others might wish to provide to platforms with substantially less private information. For some individuals, the obligation to provide sensitive information, such as credit card numbers or private addresses, might determine the choice to not participate in the sharing economy.

While data exchange is essential for the functioning of most platforms, we think that the different privacy sensitivities of users could be an opportunity for sharing platforms to reflect on whether they could offer services catering more explicitly to users with higher concerns. This could lead to the participation of current non-users and to a more engaged participation of existing users who have higher privacy concerns.

We see the following opportunities:

- All existing platforms should make sure that sensitive information, such as user profiles, names, phone numbers, and addresses is exclusively available to other users only once a sharing agreement has been reached between the two parties.

- When it comes to face-to-face interaction, platforms could implement ways for both consumers and providers to signal, for example, that they do not wish to talk to drivers/passengers during a shared ride.

- New platforms might decide to cater exclusively to individuals having higher privacy sensitivities, for example allowing users to employ a username rather than a real name, and minimizing the request for private information. This would lead to services that are more similar to traditional alternatives to the sharing economy (with minimal interaction between consumer and provider), which could however attract current non-users who feel like a traditional sharing economy experience leads to excessive exposure.

We understand that data collection and analysis is essential for the survival of sharing platforms. However, platforms should take into account that users evaluate the benefits of their participation against what they perceive as risks. Working on the perception of such risks, for example by providing more privacy-sensitive alternatives, could improve the trust of users towards the platforms, and therefore enrich their experience and make their participation more worthwhile. 


\section{Recommendations for Educators}

\section{Educators should prioritize digital literacy}

In order to become participants of the sharing economy, citizens need to be skilled users of the Internet. This cannot be achieved without significant efforts towards making digital literacy a priority for educators. In fact, as the majority of European citizens is now capable of accessing the Internet through their mobile phone, and therefore able to download the apps necessary to participate in the sharing economy, it is crucial that education about digital technologies becomes central in schools and universities.

We recommend that digital literacy is re-interpreted so that the focus of educators not only falls on the ability to use technology and participate in digital communication, but also includes informing current and prospective users on how their data is collected, how it can be used, and what strategies can be put in place so as to achieve a wished level of privacy control. Particularly for younger users, who might primarily access platforms through mobile devices, educators pay attention that information is provided on the following topics:

- Platform algorithms: What are the pros and cons of personalization? What are the traces users leave when browsing websites or accessing social media? How can they impact their experience of the Internet?

- GPS/Location data: How do apps communicate via GPS? What type of data does a mobile device communicate, just by being in a specific position? How can the exchange of locationbased data be limited?

- Phishing and identity theft: How can users spot a phishing email? What are safe behaviors to keep? Who should be contacted in case of identity theft?

Education about digital literacy, especially covering skills that are required by mobile technology cannot stop at schools and formal education. Older users, and especially those who start from a lower level of digital skills, should be informed about the opportunities and the risks of digital communication so that they can participate in the sharing economy with safety and awareness. We recommend that educators formulate campaigns that can reach these users through their workplace or through their local communities. 


\section{Educators should focus on users' rights}

Societies like the ones we find in most European cities require data privacy and data rights to be at the center of education, both through formal school programs and through campaigns targeting adults and other parts of the population. The emergence of the sharing economy, especially as it establishes itself as a source of additional income for citizens, makes this task even more crucial for educators. In fact, lack of information on data rights around the sharing economy can damage users in several ways:

- They might share more data than what requested, exposing themselves to frauds and risks.

- They might perceive sharing as excessively risky and therefore only participate as consumers.

- They might perceive the sharing economy as dangerous for their data and personal safety, and therefore decide to not participate.

We therefore believe educators have an essential role in facilitating conscious and safe participation in the sharing economy for both consumers and providers.

To address concerns relating to the treatment of data by sharing platforms, we suggest educators integrate information on data collection in their school curricula, especially towards high school and higher education, focusing on students who are of age to participate in the sharing economy. Education should focus on existing European and Member State regulations around how long private data can be stored by platforms and what rules exist around acquisition of platform data. While we think this type of education can and should start from schools, we also believe it should include adults and focus in particular on potential sharing economy users who are particularly privacy conscious, such as older users who might not feel like they have sufficient privacy skills. Educators should think of campaigns to reach such potential users and provide them with information that could mitigate their concerns.

We think two essential topics should be covered by educators approaching the sharing economy:

- The right to opt-out: Can users withdraw from a sharing platform? Can they cancel their account? Who has rights over their data when they do? Educators should provide guidelines helping users understand what happens should they decide to no longer participate.

- Algorithms and personalization: Even when sharing platforms are sometimes reticent about the rules that determine rankings, it is important for current and prospective users to be aware of how algorithms work and how their personal data affects the information they have access to. Educators should include personalization in the topics they cover within data rights. 


\section{Educators should promote safer participation}

The focus of educators, while providing information about the sharing economy, should be targeted towards promoting a wider, more informed, and ultimately safer participation of both consumers and providers of platforms, either current or prospective.

Since a significant route of access to the sharing economy happens through mobile phones and other portable devices, a first approach is to promote app-based digital literacy. This means:

- Informing the public about mobile-based data collection, for example through location sharing.

- Educating citizens about the potential risks of shared network practices.

The objective should be to put users in the position of being able to evaluate independently whether their behavior is exposing them to a level of risk they feel comfortable with, or feel like is worth the service they receive in return.

With the same aim in mind, that is, helping users make informed choices, we believe another important aspect of digital interactions should be covered by educators, that is, the inter-platform transfer of personal data. In practice, they should focus on the privacy consequences of signing into a sharing economy platform using data from one's email or through their username and password combination from a social network site. As single-access is perceived to be convenient by users and therefore widely employed, we believe it's the educators' responsibility to make sure that consumers and providers of the sharing economy also know what the consequences are for their data.

One last theme that should be covered by educators in order to promote safer sharing economy interactions concerns mitigating peer-related privacy concerns. This is done by informing the public about the limits of information that a user can request from another user, whether providers or consumers. Educators should also cover existing regulation about private data being shared on public or semipublic channels, for example social network sites, and advise users on what to do should that violate their privacy boundaries. 


\section{Educators should approach local differences}

Sensitivity to privacy, especially when it comes to limits to private possessions and data, varies significantly across countries and cultures. This can depend on several factors, such as:

- (Lack of) Local regulation.

- Traditional or religious customs.

- Differences in technological diffusion and literacy.

This can give rise to challenging differences when privacy definitions have to be found and applied, across different countries. We recommend that this aspect is not overlooked when thinking of what educators can do to promote a safer and more equitable participation to the sharing economy.

We think further research should be put into understanding the different cultural sensitivities to privacy across Europe,and that such research should inform the way privacy and data rights are taught both within and outside formal education. We advocate a dialogue between academia, education, and communities, so that not one approach is formulated for privacy and local preferences are accommodated for. Privacy education cannot happen without consideration for the sensitivity of citizens.

This matter becomes particularly important when approaching users who are more vulnerable to be discriminated against within the sharing economy, or who are simply more likely to suffer from privacy violations, either digitally or in real life. A privacy education that is heavily grounded in cultural, religious and geographical sensitivities to confidential information is more likely to change risky behaviors and promote well-informed ones. 


\section{Educators should lead by example}

Part of the education that can lead to a more aware, safer, and enthusiastic participation to the sharing economy starts with a consideration of sharing as a value. We think that educational institutions have a responsibility in making sure that some of the potential behind the sharing economy, which is in the increased sustainability of a system where peers contribute their own property for the use of other peers, is not only communicated, but embraced.

Since research centers, schools, and universities represent environments where the sharing of knowledge happens, we recommend that they become real spaces of sharing cultures. They should promote, for example:

- An open approach to data, which might include public availability of the results of surveys ran across students as well as faculty, as well as commitment to data openness and transparency.

- An unbiased education to privacy, where current and future users are informed about the risks of operating online, taking into consideration the benefits of technology and its presence in everyone's lives.

- An education about the sharing economy, which covers the full spectrum of available platforms, focusing in particular on those that do not include business transactions, and that can therefore be more accessible and most useful for younger users.

We believe that schools and universities should promote openness within their premises and become spaces where sharing cultures are encouraged and can be exported. 


\section{Recommendations for Policy Makers}

\section{Policy makers should regulate data collection by platforms}

The regulation of how platforms collect and use consumer data should represent a priority for regulators, especially at a European level. In fact, the establishment of clear rules around the amount of information that can be collected by individual platforms is essential in order to achieve three main goals:

- Avoid market imbalances, such as monopolies based on the ownership of a larger amount of data.

- Promote more aware interactions between platforms and users, where the latter know what type of information can be requested and can make more informed decisions around whether to provide it.

- Provide homogeneous rules for organizations that are often multinationals, operating over multiple fields.

We have identified three areas on which data collection regulation should focus:

- The amount and types of user data that platforms can collect: Policy makers should regulate the kind of personal information that platforms can collect, as well as how it should be stored, so as to guarantee security for all participants.

- How long consumer data should be kept on the platform's servers: Giving consumer data an 'expiration date' protects the long-term privacy of users, at the same time guaranteeing platforms' data use for such time.

- The extent to which such data is exchanged or commercialized across platforms: This could set a limit to the accumulation of user data by platforms external to the sharing economy, such as social network sites.

Those three areas represent the priority list for policy makers who wish to regulate their data collection and use. In the following section, we elaborate on how such a goal can be achieved. 


\section{Policy makers should deal with internationality}

At least for large platforms within the sharing economy, internationality is such a prominent feature within their business models that one individual legal system is hardly capable of providing sufficient regulation. It has been argued that sharing platforms would benefit the most from 'borderless', crossnational legal systems. However, such systems hardly exist at the present time, and - some argue might present more challenges than benefits for all other parties involved, such as citizens and governments.

At a European level, such a perspective can be thought of as somewhat easier to obtain, if regulators focus on promoting transparency at all levels of participation, starting from the citizens of member states, to their governments, and to platforms operating on their territory. Important steps are already being undertaken to make uniform legal procedures within the European Union. The EU data protection ordinance for 2018 can indeed be considered a milestone concerning this point. However, more steps could be necessary, considering that many of the sharing economy platforms are either based in the United States, or maintain important commercial connections overseas.

When thinking about platform regulation, specifically dealing with internationality, the following themes should be included:

- Location of servers and compliance to local rules: Servers should meet certain standards. In particular, platforms should communicate where their servers are located. In case they are not in the same country where a platform has its headquarters, regulators should ask for proof of how the company plans to respond to local data protection laws.

- Local data collection and storage: Operating in different countries will lead to different approaches to data collection and storage. Platforms should provide information around the different types of data they collect and prove that they conform to the rules the collection and storage of such data.

- Location of additional services: Platforms usually also make use of additional support systems, such as customer care or newsletters. Regulators should care that also providers of additional services respect local regulations around data collection and storage.

- Mention of all countries involved in data collection and processing: As different countries have different data protection laws, it is important that platforms disclose where data is collected and handled. 


\section{Policy makers should deal with complexity}

The term 'sharing economy' includes a number of organizations which differ by size, scope, and oftentimes operate across several types of business. As such, they tend to collect, and employ, very different types of data. It is important for policy makers to take this into consideration.

In order to provide easier regulation, policy makers should consider incentivizing a system which classifies platforms by size and defines, per each size level, specific guidelines for data processing that could help companies fulfill the requirements of the law. The kind of data companies within the sharing economy have to deal with may differ. As the sharing economy is such a fast-moving industry, it might be too challenging for regulators to account for each individual industry sector when formulating rules, however keeping the inherent complexity of the sharing economy in mind can help establishing rules that can be applied across sectors. This could also help regulation to become more transparent and easier to understand for each platform.

When thinking of platform regulation specifically dealing with operations in multiple businesses, the following themes should be included:

- Establish responsibility for data use/management: As different teams might access data at different times, it is important for regulators to make sure platforms have ways of reporting who handled data in every different phase of operations.

- Limit accessibility of data: Regulators should also set limits to the amount of data that is collected in connection with operations. They should also consider putting limits on who should be able to access the private data of users.

- Data collection and storage: When regulating how long data is allowed to remain on a platform's servers, regulators should keep in mind that different rules might exist depending on the level of sensitivity of data involved in different operations. It is thus advisable to provide guidelines on how to proceed within different scenarios. 


\section{Policy makers should ease communications and establish responsibilities}

Policy makers have an important role in establishing and incentivizing communications between platforms and users. This task is of crucial importance because the two groups have different interests and different stakes when it comes to data. Platforms need user data to function and users might have concerns about how their data is used. Policy makers should keep both needs in mind and regulate while trying to negotiate an approach that matches both interests.

Policy makers should focus, in particular, on facilitating communications between users and platforms on the following themes:

- Data deletion: Users of sharing platforms must be granted the right to delete their profile and connected data. Platforms should communicate clearly and publicly how doing this is possible.

- Use of cookies: A cookie is a message given to a browser by a web server. The browser stores such message in a text file. The message is then sent back to the server each time the browser requests a page from the server. The main purpose of a cookie is to identify users and possibly prepare customized web pages for them. When websites use cookies they should be forced to inform the user.

- Dealing with third parties: Platforms usually offer users the possibility to have public profiles, where their data can be viewed and collected by third parties. Policy makers should regulate the extent to which this is possible and establish clear communication towards users.

In providing support to handle the relationship between users and platforms, policy-makers should also help in the establishment of reciprocal responsibilities. For example, in the case of privacy violations carried out by a provider, it can be difficult to assess whether the platform should also be held responsible for the user's misconduct, given that the relationship between the two is not one of traditional employment. Policy makers should think of providing rules aimed at establishing accountability. This should help because it can support consumers in reporting claims of misconduct, as they would know exactly who is to hold responsible, thereby improving their sense of safety. It can also help the feeling of security of providers, who can feel more supported by the platform in case of privacy-related incidents. 


\section{Policy makers should regulate data collection by peers}

While policy makers should indeed focus on platforms' collection and use of data, they should also pay attention to data collection carried out by peers, such as providers asking consumers for additional information, or consumers collecting pictures and other types of data about the providers they have chosen or have been assigned to.

Just like the responsibility of peers/platforms in cases of wrongdoing by a user should be established, we believe that setting clear rules around what private data should be available by users of a platform is essential for the overall wellbeing of the sharing economy. In practice, this means providing legal guidance on a few issues:

- What data, additional to what is requested to set-up a profile, are users allowed to request from other users? For example, should providers ask for passports or other identifying documents of their consumers? Could consumers ask other consumers for the addresses and phone numbers of providers they have previously interacted with?

- What uses of such data are to be considered fair? For example, should providers be allowed to promote themselves through consumers' private phone numbers or social media profiles? Should consumers be able to access providers' names and identifying data before they enter into a sharing agreement with them?

- How and how long should peer data be kept? Just as policy makers should enforce existing regulation on data storage by providers, a similar approach should be put in place for peers.

In general, we suggest that such matters are managed by offering guidelines for both providers and consumers, which can help homogenize the different approaches to data collection by peers. The sharing economy includes a very wide array of different services, but we think there is a value in unifying, as much as possible the existing rules for the data that can be accessed and collected by individuals on the platform. This can help minimizing the opportunities for data misuse, and the potential for users to stalk, mistreat, or otherwise exploit the data of other users. 


\section{Conclusions}

In order for the sharing economy to function properly, private data from users is necessary so that providers and consumers can be matched, as well as to provide well-functioning and safe platforms that can sustain and facilitate interactions. The information users share with platforms, however, includes several elements that could be considered sensitive or confidential, such as addresses, phone numbers, credit card details, and intellectual property. Such information is disclosed voluntarily and is, in many ways, a currency users spend to guarantee their access. This, however, doesn't exclude that users might not feel comfortable with providing such amounts of personal information, or that they might worry about privacy invasions by the platforms or by peers.

We feel that, because of the sharing culture behind them, sharing platforms should encourage a system where enough data is provided so that platforms can function safely, but also where safety, legality, and respect of individual boundaries is provided.

We believe that, while platforms have important responsibilities in making sure that users are listened to, especially when they have privacy concerns, all the stakeholders involved should have a role. Policy makers should have the best interests of users in mind and should regulate how data is collected and stored. Both categories of users, providers and consumers, should stay informed and put effort into respecting the boundaries set by their peers. Educators should provide support and literacy, which can help users make more informed decisions around their data sharing habits and achieve a more involved and enthusiastic level of participation.

As the sharing economy faces challenges which might alter its nature, highlighting its commercial value over its social element, we think that all stakeholders should be involved, so that a healthy system is maintained, which carries users at its very center. 


\section{References}

Andreotti, A., Anselmi, G., Eichhorn, T., Hoffmann, C. P., Jürss, S., \& Micheli, M. (2017a). European Perspectives on Participation in the Sharing Economy. SSRN Electronic Journal. Retrieved from https://papers.ssrn.com/sol3/papers.cfm?abstract id=3046550

Andreotti, A., Anselmi, G., Eichhorn, T., Hoffmann, C. P., Jürss, S., \& Micheli, M. (2018). Recommendations for the Sharing economy: Increasing Participation. SSRN Electronic Journal. Retrieved from ps2share.eu

Andreotti, A., Anselmi, G., Eichhorn, T., Hoffmann, C. P., \& Micheli, M. (2017). Participation in the Sharing Economy. SSRN Electronic Journal. Retrieved from https://papers.ssrn.com/sol3/papers.cfm?abstract id $=2961745$

Newlands, G., Lutz, C., \& Fieseler, C. (2017a). Power in the Sharing Economy. SSRN Electronic Journal. Retrieved from https://papers.ssrn.com/sol3/papers.cfm?abstract id=2960938

Newlands, G., Lutz, C., \& Fieseler, C. (2017b). European Perspectives on Power in the Sharing Economy. SSRN Electronic Journal. Retrieved from https://papers.ssrn.com/sol3/papers.cfm?abstract id $=3046473$

Newlands, G., Lutz, C., \& Fieseler, C. (2018). Recommendations for the Sharing Economy: (Re-)Balancing Power. SSRN Electronic Journal. Retrieved from https://papers.ssrn.com/sol3/papers.cfm?abstract id=3106584

Ranzini, G., Etter, M., Lutz, C., \& Vermeulen, I. E. (2017). Privacy in the sharing economy. SSRN Electronic Journal. Retrieved from https://papers.ssrn.com/sol3/papers.cfm?abstract id=2960942

Ranzini, G., Etter, M., \& Vermeulen, I. E. (2017). European Perspectives on Privacy in the Sharing Economy. SSRN Electronic Journal. Retrieved from https://papers.ssrn.com/sol3/papers.cfm?abstract id $=3048152$

Ranzini, G., Newlands, G., Anselmi, G., Andreotti, A., Eichhorn, T., Etter, M., Hoffmann, C. P., Jürss, S., \& Lutz, C. (2017). Millennials and the Sharing Economy: European Perspectives. Retrieved from https://papers.ssrn.com/sol3/papers.cfm?abstract id=3061704

Stanoevska-Slabeva, K., Lenz-Kesekamp, V., \& Suter, V. (2017). Platforms and the Sharing Economy: An Analysis. Report for the EU Horizon 2020 Project Ps2Share: Participation, Privacy, and Power in the Sharing Economy. Retrieved from https://www.bi.edu/globalassets/forskning/h2020/ps2share platform-analysis-paper final.pdf

Stanoevska-Slabeva, K., Lenz-Kesekamp, V., \& Suter, V. (2018). Design Guidelines for Platforms in the Sharing Economy (working title). Report for the EU Horizon 2020 Project Ps2Share: Participation, Privacy, and Power in the Sharing Economy, forthcoming. 